\title{
Proyección social: reflexión sobre una experiencia virtual con adolescentes en época de pandemia en Sucre y Bolívar, Colombia
}

\author{
Claudia Yaneth Martínez-Mina \\ Magister en Ciencias del Movimiento Humano, Trabajadora social \\ Corporación Universitaria del Caribe-CECAR. Sincelejo, Colombia \\ https://orcid.org/0000-0003-1093-9161・cwa0708@hotmail.com
}

\begin{abstract}
Resumen
Este artículo muestra la experiencia de campo con estudiantes de Trabajo Social de sexto y séptimo semestre de la Corporación Universitaria del Caribe, CECAR. Como una de las estrategias de proyección social del programa académico, el estudiantado ejecutó un ejercicio de Trabajo Social de grupo de forma virtual con adolescentes de instituciones educativas ubicadas en los departamentos de Sucre y Bolívar, Colombia. El ejercicio tuvo como objetivo ofrecer apoyo social a adolescentes en tiempos del COVID-19, y duró aproximadamente 4 meses entre la planeación y la finalización del mismo. A partir de este ejercicio práctico, el estudiantado de Trabajo Social desarrolló habilidades y competencias relacionadas con el trabajo en equipo, la creatividad, la innovación, y el manejo de la frustración. Finalmente, se muestran las reflexiones que surgieron a partir de esta experiencia práctica, considerando 4 elementos fundamentales: la conectividad, la participación, la interacción y la creatividad.

Palabras clave: Trabajo Social de grupo; Virtualidad; Pandemia; Adolescentes; Currículo.
\end{abstract}

Recibido: 06/07/2021 | Aprobado: 18/10/2021 | Publicado: 01/01/2022

(1)(2) Esta obra está bajo una Licencia Creative Commons Atribución-NoComercialCompartirIgual 4.0 Internacional.

Financiación o proveniencia del artículo: Este artículo de reflexión surge como resultado del ejercicio práctico realizado con estudiantes de Trabajo Social del segundo semestre del 2020 en los cursos Grupo y Comunidad I y II.

¿Cómo citar este artículo? / How to quote this article?

Martínez-Mina, C. Y. (2022). Proyección social: reflexión sobre una experiencia virtual con adolescentes en época de pandemia en Sucre y Bolívar, Colombia. Prospectiva. Revista de Trabajo Social e intervención social, (33), 99-115. doi: 10.25100/ prts.v0i33.11435.

e-ISSN: 2389-993X • https:/ / doi.org/10.25100/prts.v0i33.11435 • Universidad del Valle - Cali, Colombia 
Martínez-Mina

\title{
Social projection: Reflexion about a Virtual Experience with Teens in Times of Pandemic in Sucre and Bolivar, Colombia
}

\begin{abstract}
This article describes the field experience with Social Work professionals in training in the sixth and seventh semester of a Social Work degree at the Corporación Universitaria del Caribe, CECAR. As one of the social projection strategies of the academic program, the students implemented a group social work experience virtually with adolescents from educational institutions located in the departments of Sucre and Bolivar, Colombia. The exercise was aimed at giving social support to adolescents in times of COVID-19, and lasted approximately four months between planning and completion. From this practical exercise, the Social Work students developed skills and competencies related to teamwork, creativity, innovation, and frustration control. Finally, the reflections that emerged from this practical experience are presented, considering 4 fundamental elements: connectivity, participation, interaction, and creativity.

Keywords: Group Social Work; Virtuality; Pandemic; Teenagers; Curriculum.

Sumario: 1. Introducción, 2. Descripción de la experiencia formativa, 2.1 El ejercicio práctico grupal a través de aplicaciones digitales de comunicación, 2.2 Contacto y caracterización grupal, 2.3 Planeación, 2.4 Ejecución, 2.5 Evaluación, 2.6 Socialización final, 3. Reflexión sobre la praxis de Trabajo Social de grupo en modalidad virtual, 3.1 Conectividad, 3.2 Participación, 3.3 Interacción, 3.4 Creatividad, 4. Conclusiones, 5. Referencias bibliográficas.
\end{abstract}




\section{Introducción}

Históricamente el método de Trabajo Social de Grupo (TSG) se evidencia como una de las estrategias implementadas desde esta disciplina en los diferentes niveles de intervención social. El programa de Trabajo Social de la Corporación Universitaria del Caribe (CECAR) dispone en su malla curricular los cursos teórico-prácticos Grupo y Comunidad I y II, los cuales están principalmente enfocados al desarrollo de competencias relacionadas con la praxis del Trabajo Social de grupo y Trabajo Social comunitario. En ese sentido, el estudiantado estará en capacidad de identificar las principales necesidades y/o problemas de un contexto social determinado, con el fin de generar procesos participativos de transformación social.

La praxis considerada como una práctica que se orienta a mejorar la sociedad, articulando de forma consciente el conocimiento con la acción (Sánchez-Vázquez, 2003), es la base fundamental de los cursos teórico-prácticos, lo que implica que el desarrollo de competencias se evidencia a partir del contacto con grupos y/o comunidades. Esta aproximación con las personas se vio afectada por la pandemia a causa del coronavirus que llegó a Colombia en marzo del 2020, situación que generó una cuarentena obligatoria en toda la población colombiana. A partir de entonces, los desafíos a nivel académico y profesional aumentaron haciendo un llamado a la innovación social y a la creatividad con relación a las formas como se realizan los procesos con los grupos y/o comunidades.

Fue necesario repensar las estrategias que se llevarían a cabo en los procesos de praxis con el estudiantado de dichos cursos, por lo que, aprovechando las bondades de la tecnología, se diseñó un ejercicio de Trabajo Social de Grupo enfocado a ofrecer apoyo social en momentos de pandemia. Los desafíos se diversificaron entre los límites de acceso a internet por parte de las comunidades y las técnicas propicias por implementar para el logro de los objetivos, teniendo en cuenta que se llevarían a cabo principalmente a través de la aplicación de mensajería WhatsApp, y por Google Meet. El ejercicio práctico estuvo contextualizado en la situación coyuntural que atravesaba el mundo, al mismo tiempo que el estudiantado se vería beneficiado tomando como referencia aquel principio básico del modelo en el Trabajo Social de Grupo de ayuda mutua: "cuando ayudas a otros te ayudas a ti mismo" (Fernández-García y López-Peláez, 2014).

Pensar en lo grupal implica traer a consideración diferentes perspectivas construidas en el campo de las ciencias sociales. Para Fernández (1989), lo grupal debe estudiarse a partir de posturas no dicotómicas ni reduccionistas, que permitan abordar la influencia de lo sociohistórico en la construcción de la subjetividad, y la de la subjetividad en lo sociohistórico. Para la autora, lo grupal y lo individual no deben verse como opuesto y contrario, sino que es indispensable reflexionar sobre la forma como los grupos constituyen un campo de mediación entre los individuos y las sociedades. Es por esto que el análisis no 
debe centrarse en los acontecimientos que surgen de las interacciones de los individuos que conforman el grupo, puesto que esta es una visión ensimismada del grupo; en su defecto, se deben tener en consideración aspectos institucionales, sociales e históricos que atraviesan a las personas que pertenecen a dichos grupos. Por consiguiente, lo grupal debe pensarse como un campo de problemáticas, a partir de epistemologías pluralistas y transdisciplinarias, que vean al grupo como un espacio de enlaces y desenlaces de subjetividades, es decir, nodos grupales que permitan un entramado de múltiples inscripciones (Fernández, 1989).

Desde un punto de vista psicoanalítico, el grupo se considera como el conjunto de relaciones internalizadas que tiene efectos intrasubjetivos en las personas que lo conforman, en donde cada persona tiene un rol que se va reajustando progresivamente en pro de la homogeneidad de una tarea. Estas características hacen parte de lo que Pichón-Riviére (1985) denomina grupos operativos. En ese sentido, los grupos aprenden, se comunican y se transforman, puesto que la dinámica en espiral que produce un cambio en el sujeto que pertenece al grupo, hace que el medio también se modifique, y de esa manera, al transformarse el medio el sujeto se modifica a sí mismo.

En concordancia con la perspectiva anterior, para García (1997), la estructura del grupo está organizada según la internalización recíproca, que, desde un punto de vista sistémico contiene una coherencia interna y una interdependencia entre los sujetos que lo constituyen. Es así como las relaciones anónimas se transforman en relaciones de interioridad al tener al otro como modelo, rival y objeto. En ese sentido, la internalización y la necesidad se consideran como el punto inicial para la interacción dentro de los grupos. Desde esta postura ocurre un proceso dialéctico entre los sujetos, puesto que estos se influyen, se determinan y se constituyen de una forma recíproca. Los cambios dentro del grupo se dan a partir de los efectos de la interacción, los cuales son tanto internos como intersubjetivos, es decir, las transformaciones internas a su vez generan cambios en lo relacional observable (García, 1997).

Finalmente, se traen a colación las posturas clásicas y tradicionales dentro del Trabajo Social, frente a lo que es el método de intervención de grupo conceptualizado de diferentes maneras, aunque con elementos comunes con relación al propósito del método. Konopka (1968), expresa que el Trabajo Social de grupo es un método que ofrece ayuda a las personas a mejorar su funcionamiento social y a enfrentarse a sus problemas personales, grupales y comunitarios, a través de experiencias constructivas de grupo. Por otro lado, Contreras de Whilelm (2003), lo considera un proceso que busca capacitar a las personas para que conozcan su realidad, y su forma de actuar dentro de la estructura social. Para esto el TSG le otorga más posibilidades a la persona de solucionar sus problemas personales, familiares, grupales y comunitarios, por medio de experiencias. Por último, los autores FernándezGarcía y López-Peláez (2014), definen el TSG como una especialidad del Trabajo Social, 
orientado a recuperar y fortalecer las capacidades sociales de las personas, aumentar su enriquecimiento personal y su funcionamiento social a través de la interacción grupal y la realización de las actividades de grupo. Al analizar cada una de estas formas de describir lo que es el TSG, se evidencia que la interacción grupal es el principal medio o mecanismo por el cual se generan esas capacidades, ayudas o capacitaciones, lo que convierte este elemento en un aspecto fundamental para que a través del método se obtengan los resultados esperados.

El desafío ante la formación de grupos para llevar a cabo cualquier intervención a partir del método de TSG, consiste en generar procesos de interacción significativos que permitan el logro de los objetivos individuales, grupales, familiares o comunitarios. En un entorno virtual, la interacción cuenta con unas limitaciones relacionadas con el tipo de comunicación que se propicia. Por ejemplo, la interacción de tipo virtual implica que la comunicación sea tanto sincrónica como asincrónica dependiendo del medio o aplicación virtual que se utilice. Asimismo, la comunicación no verbal en espacios virtuales se limita al uso de emojis, imágenes, stickers, entre otros, y la interacción se da solamente cuando existe una comunicación explícita. Es decir, si alguna persona está en un grupo de WhatsApp, pero nunca escribe, no hay interacción recíproca con esa persona. Lo contrario sería si el grupo estuviera en contacto presencial, pues, aunque la persona no se exprese abiertamente, la comunicación no verbal siempre está presente (mediante miradas, gestos, posturas).

Otro concepto importante en este ejercicio es el de apoyo social, puesto que el objetivo estuvo enfocado a ofrecer este tipo de ayuda. El apoyo social es visto como la ayuda recibida de distintas redes sociales, tanto formales como informales (De Federico de la Rúa, 2008). En el caso del ejercicio práctico en mención, el apoyo social estuvo enfocado a lo emocional partiendo de valores como la empatía, el cuidado, el amor y la confianza. Esto principalmente para el establecimiento de los objetivos propuestos, tomando como referencia la situación que enfrentaba la mayoría de la población, derivada de los cambios significativos en las relaciones interpersonales y la cotidianidad en época de pandemia y confinamiento.

El uso de plataformas virtuales en la práctica de Trabajo Social para crear condiciones de interacción entre personas que pasan por las mismas problemáticas, no es resultado de esta época de pandemia; existen diversas experiencias desde la profesión a través de entornos virtuales. Por ejemplo, la implementación de foros de salud mental en el que interactúan personas que se enfrentan a problemas como la depresión, la esquizofrenia y la bipolaridad, permitió reducir sentimientos de soledad e impotencia en las personas participantes, así como ofrecer acompañamiento de iguales y relaciones de ayuda mutua (Miranda-Ruche, 2016). De igual forma, algunas experiencias muestran que el Trabajo Social online a partir de una visión funcionalista de adaptación al individuo, permite informar sobre recursos existentes, fomentar los Derechos Humanos, mejorar las relaciones grupales 
y familiares, ofrecer apoyo moral, prevenir conductas adictivas, en fin, prevenir situaciones de exclusión social a través de diversas herramientas virtuales como chats, foros de discusión, plataformas web 2.0 entre otras (Caravaca-Llamas, 2014). Fernández-García y López-Peláez (2014, p. 143) expresan que, las nuevas tecnologías y el internet posibilitan la construcción de espacios de apoyo hacia los ciudadanos, para viejos problemas como la violencia de género o nuevos como las infoadicciones

Teniendo en cuenta que el mundo no se detuvo y las instituciones educativas continuaron impartiendo clase a partir de la virtualidad, la beneficiaria del proceso práctico fue la población adolescente, tomando del mismo modo la virtualidad como medio para el intercambio interpersonal y el contacto con las personas. Se crearon enlaces con las instituciones educativas pertenecientes a la zona rural y urbana de los departamentos de Sucre y Bolívar, específicamente de los municipios de origen del estudiantado que se encontraban cursando en el semestre 2020-2 los cursos Grupo y Comunidad I y II. De igual manera, se involucraron como beneficiarios del ejercicio, estudiantes de primer semestre del programa de Trabajo Social de la Corporación Universitaria del Caribe. En la ejecución participaron 97 profesionales de Trabajo Social en formación de sexto y séptimo semestre, y se crearon 20 grupos virtuales con el objetivo de ofrecer apoyo social a adolescentes en tiempos del COVID-19. El ejercicio duró aproximadamente 4 meses entre la planeación y la finalización del mismo.

\section{Descripción de la experiencia formativa \\ 2.1 El ejercicio práctico grupal a través de aplicaciones digitales de comunicación}

Existen unos procesos subyacentes a los diferentes métodos de intervención social sean estos de tipo individual, grupal o comunitario, partiendo del estudio, transcurriendo por la planeación, la ejecución y la evaluación (Ander-Egg, 2009). Los anteriores procesos no son lineales sino dinámicos, por lo que cada uno al mismo tiempo se encuentra en todos. Antes de mencionar cada una de las fases desarrolladas en el ejercicio práctico es importante resaltar que fue necesario realizar un contacto previo con las directivas de las instituciones educativas en las cuales se llevaría a cabo el ejercicio. Para dicho procedimiento se envió un correo electrónico de agradecimiento, al mismo tiempo que mostraba el objetivo y cada una de las fases que serían implementadas. Para llevar a cabo el ejercicio práctico a partir del método de TSG, se implementó una metodología diseñada para tal fin, tomando como referencia los procesos subyacentes que acabamos de mencionar los cuales se detallan a continuación. 


\subsection{Contacto y caracterización grupal}

En esta primera fase se buscó conocer aspectos de tipo individual, y el contexto sociofamiliar del estudiantado. También fue indispensable identificar las condiciones de conectividad para definir las herramientas virtuales a utilizar. Para dicho fin, cada grupo de trabajo conformado por profesionales de Trabajo Social en formación, de sexto y séptimo semestre, solicitaron al enlace de la Institución Educativa, I.E, (rectores/as, coordinadores/as, docentes) la información de contacto que, en este caso, fueron los números de teléfono de cada uno/a de los/as estudiantes seleccionados. En este primer contacto fue necesario solicitar el consentimiento informado al acudiente por tratarse de menores de edad, quienes a través de la manifestación verbal aceptaron o rechazaron la participación de cada adolescente en el proceso. Únicamente se tuvo en cuenta para el ejercicio a los y las estudiantes que tuvieron previamente el consentimiento informado de sus padres, madres y/o cuidadores.

\subsection{Planeación}

En el transcurso de las clases remotas y partiendo del principio de participación en las aulas, durante un mes aproximadamente el estudiantado indagó en los temas correspondientes al proyecto de vida, el apoyo social, y la prevención de la violencia de género. De esa manera, profundizaron en cada temática general, de acuerdo con la preferencia de cada grupo, reconociendo los elementos teóricos necesarios a tener en cuenta para el trabajo con adolescentes. Posteriormente, se realizó un análisis de contenido con toda la información obtenida por el estudiantado, lo que dio como resultado unas categorías o temas específicos a ser tratados en cada una de las sesiones. De igual forma, en algunas clases se dialogó sobre diferentes metodologías y actividades que podrían implementarse de forma virtual a través de WhatsApp y otras aplicaciones de videoconferencias. Al finalizar el proceso de indagación se escogieron los siguientes temas distribuidos en 10 sesiones (Ver Tabla 1): 
Martínez-Mina

Tabla 1. Planeación de las sesiones relacionadas con los temas generales de intervención.

\begin{tabular}{|c|c|c|c|c|c|c|}
\hline $\begin{array}{c}\text { Temática } \\
\text { general } \\
\text { del } \\
\text { ejercicio }\end{array}$ & \multicolumn{2}{|c|}{ Grupo: proyecto de vida } & \multicolumn{2}{|c|}{ Grupo: apoyo social } & \multicolumn{2}{|c|}{ Grupo: violencia de género } \\
\hline $\begin{array}{c}\text { \# de } \\
\text { sesión }\end{array}$ & Tema & $\begin{array}{l}\text { Nombre de } \\
\text { la actividad }\end{array}$ & Tema & $\begin{array}{l}\text { Nombre de } \\
\text { la actividad }\end{array}$ & Tema & $\begin{array}{c}\text { Nombre de la } \\
\text { actividad }\end{array}$ \\
\hline 1 & $\begin{array}{l}\text { Inicio y } \\
\text { presentación }\end{array}$ & $\begin{array}{l}\text { Nuestro } \\
\text { primer } \\
\text { encuentro }\end{array}$ & $\begin{array}{l}\text { Inicio y } \\
\text { presentación }\end{array}$ & $\begin{array}{l}\text { Nuestro } \\
\text { primer } \\
\text { encuentro }\end{array}$ & $\begin{array}{l}\text { Inicio y } \\
\text { presentación }\end{array}$ & $\begin{array}{l}\text { Nuestro } \\
\text { primer } \\
\text { encuentro }\end{array}$ \\
\hline 2 & $\begin{array}{l}\text { Historia } \\
\text { personal }\end{array}$ & $\begin{array}{l}\text { Reconozco } \\
\text { mi historia }\end{array}$ & $\begin{array}{l}\text { Emociones y } \\
\text { sentimientos }\end{array}$ & $\begin{array}{l}\text { Así me } \\
\text { siento }\end{array}$ & $\begin{array}{l}\text { Feminidades y } \\
\text { masculinidades }\end{array}$ & $\begin{array}{l}\text { ¿Qué es ser } \\
\text { mujer? ¿Qué } \\
\text { es ser hombre? }\end{array}$ \\
\hline 3 & Autoestima & Soy calidad & Autoestima & Soy calidad & Autoestima & Valgo la pena \\
\hline 4 & $\begin{array}{l}\text { Visión y } \\
\text { misión }\end{array}$ & Mi legado & $\begin{array}{l}\text { Visión y } \\
\text { misión }\end{array}$ & Mi legado & $\begin{array}{l}\text { Presión sobre el } \\
\text { cuerpo }\end{array}$ & $\begin{array}{l}\text { Me acepto } \\
\text { como soy }\end{array}$ \\
\hline 5 & $\begin{array}{l}\text { Talentos y } \\
\text { habilidades }\end{array}$ & $\begin{array}{l}\text { Creo en mi } \\
\text { potencial }\end{array}$ & $\begin{array}{l}\text { Estrés y } \\
\text { ansiedad }\end{array}$ & $\begin{array}{l}\text { Conectándo } \\
\text { me con mi } \\
\text { ser }\end{array}$ & $\begin{array}{l}\text { Acoso sexual } \\
\text { escolar }\end{array}$ & $\begin{array}{l}\text { Dile NO al } \\
\text { acoso sexual }\end{array}$ \\
\hline 6 & $\begin{array}{l}\text { Oportunidades } \\
\text { del entorno }\end{array}$ & $\begin{array}{l}\text { Vale la } \\
\text { pena } \\
\text { intentarlo }\end{array}$ & $\begin{array}{l}\text { Historia } \\
\text { familiar }\end{array}$ & $\begin{array}{l}\text { Lazos que } \\
\text { no se } \\
\text { rompen }\end{array}$ & Noviazgo & $\begin{array}{l}\text { Te quiero, } \\
\text { pero no más } \\
\text { que a mi vida }\end{array}$ \\
\hline 7 & $\begin{array}{l}\text { Crecimiento } \\
\text { personal }\end{array}$ & $\begin{array}{l}\text { Sigo } \\
\text { creciendo }\end{array}$ & $\begin{array}{l}\text { Hobbies y } \\
\text { tiempo libre }\end{array}$ & $\begin{array}{l}\text { Mi tiempo } \\
\text { libre mi } \\
\text { tiempo sano }\end{array}$ & $\begin{array}{l}\text { Violencia en el } \\
\text { noviazgo }\end{array}$ & $\begin{array}{l}\text { Libres en el } \\
\text { amor }\end{array}$ \\
\hline 8 & Sexualidad & Sin tabúes & Sexualidad & Sin tabúes & Sexualidad & Sin tabúes \\
\hline 9 & $\begin{array}{l}\text { Emociones y } \\
\text { sentimientos }\end{array}$ & $\begin{array}{l}\text { Así me } \\
\text { siento }\end{array}$ & $\begin{array}{l}\text { Internet y } \\
\text { redes } \\
\text { sociales }\end{array}$ & $\begin{array}{l}\text { Las trampas } \\
\text { del Sexting }\end{array}$ & Sexting & $\begin{array}{l}\text { Las trampas } \\
\text { del sexting }\end{array}$ \\
\hline 10 & $\begin{array}{l}\text { Clausura y } \\
\text { evaluación }\end{array}$ & $\begin{array}{l}\text { Nuestro } \\
\text { último } \\
\text { encuentro }\end{array}$ & $\begin{array}{l}\text { Clausura y } \\
\text { evaluación }\end{array}$ & $\begin{array}{l}\text { Nuestro } \\
\text { último } \\
\text { encuentro }\end{array}$ & $\begin{array}{l}\text { Clausura y } \\
\text { evaluación }\end{array}$ & $\begin{array}{l}\text { Nuestro } \\
\text { último } \\
\text { encuentro }\end{array}$ \\
\hline
\end{tabular}

Fuente: elaboración propia. 


\subsection{Ejecución}

Posterior a las llamadas de caracterización realizadas a cada adolescente que participaría en el proceso, cada equipo de trabajo conformado por estudiantes de Trabajo Social procedió a la creación del grupo de WhatsApp desde el cual se llevarían a cabo las actividades con los y las adolescentes focalizadas. Algunos equipos de trabajo implementaron las actividades utilizando más de una aplicación, por ejemplo, Meet y Zoom, asimismo el diseño y creación de las actividades fue libre, propiciando de esa manera el desarrollo de su creatividad como competencia transversal. Esa planeación de cada sesión se plasmó en el formato de planeación de actividades y/o procesos formativos. Posterior a la realización de la actividad virtual, cada estudiante de Trabajo Social de manera individual diligenció el diario de campo relacionado con su experiencia particular en esa sesión. El día y hora de cada sesión fue concertado con los y las adolescentes desde el grupo de WhatsApp creado, intentando no coincidir con los horarios de clase $\mathrm{u}$ otras actividades académicas tanto de los y las adolescentes como de los grupos de estudiantes de Trabajo Social. De esa manera, los encuentros se desarrollaron con una frecuencia semanal y una duración máxima de una hora.

\subsection{Evaluación}

Al finalizar cada sesión, se aplicó una encuesta de satisfacción con relación a la actividad, siendo este un mecanismo de seguimiento adaptado a la virtualidad y a la edad de la población beneficiaria. Esa encuesta de satisfacción fue diseñada de forma creativa y participativa por los cursos de sexto y séptimo semestre de Trabajo Social, partiendo de un modelo implementado en los ejercicios prácticos desarrollados anteriormente de manera presencial en el curso Grupo y Comunidad II; la nueva modificación incluyó el uso de emojis de WhatsApp. Finalizado el ejercicio práctico, se evaluaron los resultados y el proceso llevado a cabo por el estudiantado de Trabajo Social, para lo cual se implementaron dos técnicas de evaluación: una para evaluar los resultados de cada miembro del grupo de adolescentes con la técnica Cómo nos sentimos (Fernández-García y López-Peláez, 2014). Y la segunda, para evaluar los resultados en términos de objetivos a través de una entrevista con preguntas abiertas, la cual fue implementada al finalizar el ejercicio práctico con la totalidad del equipo de trabajo. 


\subsection{Socialización final}

Finalmente, los y las estudiantes de sexto y séptimo semestre de Trabajo Social socializaron su ejercicio grupal a través de una retroalimentación en la clase remota. En ese espacio fueron discutidos los aprendizajes significativos, los aspectos para mejorar, y las recomendaciones para futuros ejercicios. Asimismo, fue enviado un informe del ejercicio práctico a los y las representantes de las instituciones educativas para dar a conocer el proceso y sus resultados.

\section{Reflexión sobre la praxis de Trabajo Social de grupo en modalidad virtual}

A partir de la socialización final llevada a cabo con estudiantes de sexto y séptimo semestre de Trabajo Social, los aprendizajes con relación a la práctica desde las herramientas virtuales en estos contextos fueron significativas, y les permitió identificar algunos desafíos que trae consigo el ejercicio profesional. A continuación, se profundiza en cuatro aspectos considerados los más relevantes con relación a la reflexión final resultado del ejercicio práctico: conectividad, participación, interacción, creatividad.

\subsection{Conectividad}

Colombia es un país en vía de desarrollo que todavía presenta grandes desafíos relacionados con el acceso a servicios básicos en su población, incluyendo la conectividad digital a través de internet. Esta situación es compartida con Latinoamérica en donde las diferencias entre la conectividad entre los hogares ubicados en la zona urbana y rural suelen ser amplias; en la población urbana aproximadamente el $67 \%$ de las viviendas urbanas tienen conexión a internet frente a un $23 \%$ de la población rural (Banco Interamericano de Desarrollo [BID], Microsoft Corporation, e Instituto Interamericano de Cooperación para la Agricultura [IICA], 2020). El acceso a internet, hasta antes de la pandemia se consideraba una necesidad latente en la población rural y suburbana, y en algunos estratos de la población urbana. De acuerdo al BID, Microsoft Corporation, e IICA (2020) en el año 2018 el 66,68\% de los hogares colombianos contaban con servicio de internet, a partir del año 2020 esta cifra aumentó a 76,47\%. Al inicio de la pandemia ocasionada por el coronavirus, la cuarentena obligatoria convirtió el servicio de internet en una necesidad ineludible y trascendental para el desarrollo de las diferentes actividades cotidianas. Colombia a través del Ministerio de Tecnologías de la Información y las Comunicaciones, se esforzaba para disminuir la brecha digital en el país. En el año 2014 el operador español de satélites Hispasat y NEC Colombia, filial de la japonesa NEC Corporation, facilitaron la instalación de 648 kioskos digitales en ocho departamentos del país (EFE News Service, 2014). En el 2018 el Gobierno colombiano y la Federación Nacional de Cafeteros (FNC) firmaron un memorando de entendimiento para "potenciar" la conectividad en zonas rurales del país" (EFE News Service, 2018). En el año 2019 la empresa estadounidense Microsoft, anunció la 
inversión de 10 mil millones de pesos para mejorar la conectividad en Colombia (EFE News Service, 2019).

A pesar de los esfuerzos realizados, la desigualdad con relación al acceso a internet se hizo visible, al ser un factor fundamental para que las personas accedan a sus derechos como el de la educación en época de pandemia. Cuando se diseñó el ejercicio práctico, la hipótesis inicial era "si están recibiendo clases de forma remota, entonces podemos hacer el ejercicio de la misma manera". A medida que se avanzaba en las fases del método, se fueron evidenciando las realidades de los y las estudiantes beneficiarios/as. Por ejemplo, recibir clase desde el celular de uno de sus dos padres, usar el internet de la persona que vive a su lado (vecinos), recargar un paquete de datos desde sus posibilidades para tener internet por momentos, recibir las clases a través de guías fotocopiadas entregadas por sus docentes cada 15 días, no poseer computador para desarrollar sus tareas, contar con una conectividad limitada que genera constantes cortes y lentitud en la comunicación, y finalmente, no contar con internet. Frente a estas dificultades el ejercicio solo se pudo realizar con las personas que podían conectarse, excluyéndose a los y las estudiantes sin acceso a internet, de esa manera, la conexión digital se convierte en un privilegio en tiempos de pandemia. Para contrarrestar esta situación, el departamento de Sucre hace parte del proyecto nacional del Ministerio de las Tics en el que serán beneficiados algunos corregimientos y veredas con internet gratuito hasta el 2031 (Ministerio de Tecnologías de la Información y las Comunicaciones [MINTIC], 2020). No obstante, en el momento de llevar a cabo el ejercicio estas estrategias apenas estaban en consideración.

La conectividad a internet estuvo ligada a otro factor fundamental: el servicio de energía. En las comunidades de Sucre y Bolívar, así como en otros departamentos de la costa, el servicio de energía sufre constantes cortes, generando mayores limitaciones para la ejecución de algunas actividades. De acuerdo a Vence-Pareja y Kammerer-Kammerer (2016), para la empresa prestadora de energía Electricaribe, los factores que inciden en que el servicio de energía en la costa de Colombia se vea afectada por diversos cortes y bajas de tensión, es precisamente la alta tasa de robo de energía que supera el 20\%, lo que al mismo tiempo genera inviabilidad financiera. Situaciones como estas de índole cultural, y que están fuertemente ligadas a los factores socioeconómicos de la zona, influyeron directamente en el trabajo práctico. Fue así como se pudo evidenciar que el ejercicio virtual de Trabajo Social de Grupo, es idóneo para ejecutarse en contextos con ciertas características sociodemográficas, pues las particularidades de cada zona influyen en que pueda llevarse a cabo de forma eficiente o no. En ocasiones, las actividades no se realizaron por las circunstancias mencionadas anteriormente, esto afectó directamente la participación en las actividades posteriores. La dinámica de grupo que se evidencia de forma presencial, fue obstaculizada en las actividades virtuales por cuestiones ligadas al acceso a los servicios públicos como el internet y la energía, y la calidad de los mismos. 


\subsection{Participación}

Considerando las dificultades de acceso a internet y la calidad del servicio de energía en los contextos de intervención, la participación en el ejercicio de Trabajo Social virtual se vio fuertemente afectada. Para el Trabajo Social de Grupo, la participación es un elemento importante en cada una de las fases y momentos del método. Esta se considera como uno de los valores que están en el centro del TSG, junto con la colectividad y la interdependencia (Ward, citado en Adams, Dominelli \& Payne, 1998). Asimismo, uno de los roles fundamentales en el/la Trabajador/a Social de grupo es fomentar la participación (VillegasCastrillo, 1993), y la tarea fundamental en la fase de conformación de un grupo radica precisamente en fomentar la participación (Sepúlveda-Cerna y Vásquez- Mendoza, 2015). En ese sentido, el estudiantado de Trabajo Social encontró su mayor desafío. No obstante, los cortes de energía y las limitaciones de acceso a internet no fueron los únicos factores que influyeron en el constante descenso de la participación durante el tiempo de ejecución de las actividades. Se pudo observar que al principio los y las participantes ingresaron a los grupos de WhatsApp, Meet y Zoom para el desarrollo de las actividades, pero paulatinamente en cada sesión el número de participantes fue disminuyendo, y esta situación no se pudo resolver. Por ejemplo, a través de WhatsApp se usaron actividades creativas usando stickers, emoticones, y memes para propiciar un ambiente de confianza con los y las participantes. No obstante, en diversas ocasiones no hubo respuesta o ningún tipo de interacción de los y las estudiantes beneficiados/as. Esta situación afectó en gran medida a estudiantes de Trabajo Social, quienes usaban su creatividad y empeño planeando las actividades semanales, para posteriormente ser ignorados/as al ejecutarlas en los grupos de WhatsApp.

En cada retroalimentación semanal dada en clase, fue fundamental motivar y propiciar ese deseo de aprendizaje en el estudiantado de Trabajo Social, trabajar con la frustración y la carga emocional que les estaba generando llevar a cabo este ejercicio. Precisamente, afrontar mayores dificultades les daba la posibilidad de desarrollar en mayor medida la creatividad viendo como un desafío aumentar la participación, pero al mismo tiempo les mostró la realidad de trabajar con grupos y comunidades cuando no existe un proceso de sensibilización previo. Sin participación, el objetivo del método de Trabajo Social de Grupo se ve restringido, pues a mayor participación mayor interacción, y cuando la interacción es constante se pueden dar los resultados esperados con relación a la ayuda mutua. La participación activa de todo el grupo es crucial para el progreso, por eso la importancia en la toma de decisiones con relación a las actividades (Villegas-Castrillo, 1993). Por ese motivo, la primera sesión estaba focalizada a conocer las preferencias de los y las beneficiarias, tomando sus preferencias como punto de partida para el diseño de las técnicas a implementarse. Finalmente, el ejercicio se llevó a cabo con altas y bajas en la participación con un promedio aproximado de la mitad de beneficiarios/as totales que aceptaron estar en el proceso. 


\subsection{Interacción}

La interacción como factor fundamental en los procesos de Trabajo Social de Grupo se manifestó de forma particular, debido a la falta de participación y a la dificultad de otras formas de intercambios que se dan en lo presencial, por ejemplo, la comunicación no verbal (saludos, sonrisas, risas, gestos, miradas, etc.). Siendo así, la comunicación se considera un elemento fundamental en el desarrollo social de las personas, en el que se pueden expresar, reconocerse a sí mismas y al/la otro/a en el proceso de interacción (Mead 1972, citado en Fernández-García y López-Peláez, 2014). Desde la virtualidad, la dimensión de la interacción que se da en los procesos de comunicación grupal, se manifestó de una forma particular, que, de alguna manera, impidió el resultado esperado. Para Fernández-García y López-Peláez (2014), la comunicación y la interacción pueden ser definidas como el “intercambio de mensajes entre las personas" (p. 38), de modo que, la comunicación es la encargada de transmitir mensajes intencionales, sean estos verbales o no verbales, y la interacción está relacionada con el envío de todo tipo de mensajes, sean estos intencionales o no, conscientes o inconscientes. En este sentido, en el desarrollo del ejercicio la no participación evidenciada a partir de la ausencia de comunicación escrita o verbal, mostró a partir de dicha interacción un mensaje de desinterés, desmotivación, e incluso, se podría pensar de miedo o timidez para expresarse dentro del grupo de WhatsApp. Lo que implica que, de alguna manera, no se dio la influencia mutua entre sus participantes, es decir "interdependencia en el intercambio de los mensajes" (Fernández-García y López-Peláez, 2014, p. 39).

Finalmente, para el estudiantado de Trabajo Social, fue evidente que generar estos procesos de intercambio y de influencia desde las plataformas tecnológicas virtuales se torna un asunto de mayor complejidad. Esto no significa que sea imposible darse, solo que, para este ejercicio práctico y teniendo en cuenta el tiempo y los recursos disponibles, no fue posible que se presentara. Para el Trabajo Social en general el contacto humano es fundamental y las estrategias tecnológicas pueden facilitar en ocasiones comunicarse y propiciar intercambios sociales, pero a partir de este ejercicio, se pudo evidenciar que la comunicación no verbal dada a través de la interacción y de los mensajes intencionales y no intencionales que surgen de ella, es limitada e impidió ofrecer el apoyo social de acuerdo a la planeación, frente a los procesos de interacción presenciales.

\subsection{Creatividad}

En el desarrollo del ejercicio profesional a veces se evidencia una fidelidad por la realización de las mismas estrategias de intervención con grupos: "charlas y talleres". La creatividad fue fundamental para propiciar la participación, despertar el interés, generar reflexión y sensibilización frente a los temas a desarrollar. Para Cebrián-Lozano (2012), un equipo de Trabajo Social que se requiere en estos tiempos de cambios, cada vez más 
exponenciales, debe estar conformado por personas que no teman afrontar nuevos desafíos, que posean ideas innovadoras, que tengan la capacidad de trabajar en equipos interdisciplinares y que estén actualizados con las nuevas tendencias. Y precisamente, esas fueron las habilidades que más tuvieron que desarrollar para llevar a cabo un ejercicio de grupo desde WhatsApp con adolescentes con el fin de ofrecer apoyo social en tiempos de pandemia. La creatividad fue una constante en cada una de las acciones que se llevaron a cabo en la intervención de grupo. A pesar de las dificultades relacionadas con los elementos mencionados anteriormente (conectividad, participación, interacción), continuaron avanzando con una visión distinta frente a lo que se acostumbra realizar, readaptando y reinventando lo que estaba establecido. La orientación estaba enfocada a salirse de lo común sin perder el objetivo de la intervención, a desarrollar ideas nuevas posibles de ejecutarse en plataformas virtuales. El estudiantado de Trabajo Social se vio obligado a desarrollar la creatividad para propiciar nuevas técnicas a partir de la virtualidad, pero, al mismo tiempo, para despertar el interés y la participación tan difícil de conseguir en el ejercicio. A pesar de los esfuerzos conjuntos, la participación no fue significativa y las actividades, aunque creativas, se llevaron a cabo la mayoría de las veces con la mitad de los y las participantes iniciales. Frente a este tema, fue positivo que el estudiantado de Trabajo Social se enfrentara a situaciones como estas, identificando así la complejidad de intervenir en sus propias realidades, y en lo importante de construir estrategias disruptivas en el ejercicio profesional.

Finalmente, a partir de la técnica de evaluación aplicada en la última sesión, se pudo evidenciar que el ejercicio fue significativo para quienes participaron de forma constante, pues sintieron que contaban con el apoyo de profesionales de Trabajo Social en formación para asuntos de índole individual como familiar. Asimismo, la creatividad de las actividades les permitió pasar momentos diferentes, que, de alguna manera, les dio alivio en medio de la cuarentena obligatoria. Usar las plataformas tecnológicas para actividades distintas a las escolares fue la principal motivación para continuar con el proceso hasta el final. En ese sentido, el ejercicio práctico brindó posibilidades de aprendizaje tanto al estudiantado de Trabajo Social que lo ejecutó como a la población adolescente atendida.

\section{Conclusiones}

El Trabajo Social de Grupo es un método que permite el desarrollo de diversas habilidades para el funcionamiento social de los individuos. En época de pandemia se tornó fundamental el apoyo social hacia la población que se encontraba en cuarentena obligatoria. Desde el programa de Trabajo Social de CECAR, y como parte de los procesos de proyección social del mismo, la intención de continuar aportando a las comunidades no se detuvo, lo que llevó a afrontar desafíos con relación a la práctica de campo de los y las profesionales de Trabajo Social en formación. A pesar de las diferentes dificultades encontradas en el proceso, el objetivo del ejercicio se cumplió en las personas que participaron activamente hasta el final. La confianza de algunos/as adolescentes con los y las estudiantes de Trabajo 
Social se manifestó cuando estos pedían consejos y recomendaciones en privado, o en espacios distintos a las sesiones. Asimismo, en la fase de finalización del ejercicio, algunos manifestaron no querer que las sesiones terminaran, pues consideraban un espacio enriquecedor para sus vidas. Esta situación fue una de las satisfacciones más grandes que el estudiantado de Trabajo Social obtuvo, pues a pesar de las dificultades evidenciadas en el desarrollo de las sesiones virtuales, vieron resultados positivos en quienes se comprometieron y fueron constantes.

El aprendizaje con relación a la praxis en el estudiantado de Trabajo Social fue significativo y coherente con el modelo pedagógico social cognitivo acogido por CECAR, sus propias comunidades fueron el principal laboratorio de práctica. Además, desarrollaron habilidades para el trabajo en equipo, e identificaron la necesidad de la creatividad en el ejercicio profesional, así como la complejidad de la realidad social. Teniendo en cuenta que estos ejercicios se llevaron a cabo en sus mismas comunidades, esto les generó esa necesidad de aportar a los cambios sociales que se requieren en las mismas, siendo ellos y ellas dinamizadores para hacerlo posible.

La importancia de conocer y reconocer las condiciones del contexto antes de aplicar una estrategia, es otro de los aprendizajes importantes que surgen del ejercicio. Aquí es importante señalar la postura de Fernández, (1989) en cuanto a concebir los procesos grupales desde un punto de vista dialéctico que permita el atravesamiento de dimensiones institucionales, sociales e históricas, y no partir de posturas reduccionistas que ven al grupo como un todo y ensimismado. Es probable que el mismo proceso de Trabajo Social de Grupo funcione de una mejor manera y obtenga mejores resultados si se aplica en un contexto que haya superado las limitaciones de acceso a internet, y con un servicio de energía de mejor calidad. No obstante, la virtualidad fue una gran aliada pues permitió continuar interviniendo en las comunidades, y al estudiantado le abrió la posibilidad de aprender a partir de la praxis.

Finalmente, como sociedad y como región es necesario reconocer las brechas de desigualdad digital, especialmente en comunidades rurales y suburbanas. Propiciar procesos de mejora frente a este servicio que hoy por hoy se ha convertido en unas de las necesidades básicas de la población, al facilitar el acceso a otros derechos como el de la educación, el trabajo, la salud, entre otros. Desde Trabajo Social se debe pensar en generar procesos de intervención cada vez más innovadores, superando las barreras de los paradigmas que se han implantado respecto a la actuación profesional. Un pensamiento creativo e innovador es uno de los mayores desafíos para quienes están insertos en esta disciplina; la invitación es a pensar diferente para obtener resultados distintos que verdaderamente aporten a la transformación de personas, grupos y comunidades. 


\section{Referencias bibliográficas}

Adams, R., Dominelli, L., \& Payne, M. (1998). Social Work: Themes, Issues and Critical Debates. New York: Red Globe Press.

Ander-Egg, E. (2009). Metodologías de acción social. Buenos Aires: Lumen.

Banco Interamericano de Desarrollo [BID], Microsoft Corporation, e Instituto Interamericano de Cooperación para la Agricultura [IICA]. (2020). Conectividad rural en América Latina y el Caribe: un puente al desarrollo sostenible en tiempos de pandemia. Recuperado de https://repositorio.iica.int/handle/11324/12896.

Caravaca-Llamas, C. (2014). Trabajo social on-line. El futuro de la intervención social. Pensamiento al margen: revista digital sobre las ideas políticas, (1), 1-17.

Cebrián-Lozano, C. (2012). Creatividad en Trabajo Social: el estímulo que necesitamos. TS nova: Trabajo Social y servicios sociales, (5), 97-102.

Contreras de Wilhelm, Y. (2003). Trabajo Social de grupo. México: PAX.

EFE News Service. (07 de Diciembre de 2018). Colombia y cafeteros firman acuerdo para llevar conectividad a zonas rurales: COLOMBIA CAFÉ. Recuperado de https:/ / www.proquest.com/docview/2151386195?accountid=34487.

EFE News Service. (09 de mayo de 2019). Microsoft invertirá 10.000 millones pesos para mejorar conectividad Colombia: COLOMBIA TECNOLOGÍA. Recuperado de https:/ / www.proquest.com/docview/2222187144 ?accountid=34487.

EFE News Service. (16 de Mayo de 2014). Española Hispasat y NEC facilitarán conectividad en zonas rurales de Colombia: ESPAÑA EMPRESAS. Recuperado de https://www.proquest.com/docview/1524875250?accountid=34487 .

Federico de la Rúa, A. de (2008). Análisis de redes sociales y Trabajo Social. Revista de Trabajo Social, 8(1), 9-21. Recuperado de http://rabida.uhu.es/dspace/bitstream/handle/10272/2172/b15500470.pdf?sequence=1.

Fernández, A. M. (1989). El campo grupal. Notas para una genealogía. Buenos Aires: Ediciones nueva visión.

Fernández-García, T., y López-Peláez, A. (2014). Trabajo Social con grupos. Madrid: Alianza Editorial.

García, D. (1997). El Grupo. Métodos y técnicas participativas. Buenos Aires: Espacio.

Konopka, G. (1968). Trabajo Social de grupo. Madrid: Euroamérica.

Ministerio de Tecnologías de la Información y las Comunicaciones [MINTIC]. (2020). A Sucre llegarán 475 Centros Digitales para transformar la vida de sus habitantes con internet gratuito hasta 2031, anunció la ministra Karen Abudinen. Recuperado de https:/ / mintic.gov.co/portal/inicio/Sala-de-prensa/Noticias/160721:A-Sucrellegaran-475-Centros-Digitales-para-transformar-la-vida-de-sus-habitantes-coninternet-gratuito-hasta-2031-anuncio-la-ministra-Karen-Abudinen.

Miranda-Ruche, X. (2016). La comunidad virtual como espacio de intervención para el Trabajo Social. Elementos para el análisis desde el ámbito de la Salud Mental. En D. Carbonero, E. Raya, N. Caparrós y C. Gimeno (Coords.), Respuestas transdisciplinares 
Martínez-Mina en una sociedad global: aportaciones desde el Trabajo Social (pp. 1-15). España: Universidad de La Rioja.

Pichón-Rivière, E. (1985). El proceso grupal. Del psicoanálisis a la psicología social (Vol. I). Buenos Aires: Ediciones Nueva Visión.

Sánchez-Vázquez, A. (2003). Filosofía de la praxis. México: Siglo XXI Editores.

Sepúlveda-Cerna, M. C., y Vásquez-Mendoza, V. A. (2015). Trabajo social con grupos: un análisis descriptivo del quehacer profesional en el área de salud mental (Tesis de pregrado). Universidad del Bío-Bío. Facultad de Humanidades y Educación. Escuela de Trabajo Social. Chillán. $\quad$ Recuperado de http://repobib.ubiobio.cl/jspui/bitstream/123456789/1815/1/Sepulveda_Cerna_Maka rena.pdf.

Vence-Pareja, J. L., y Kammerer-Kammerer, M. G. (2016). Crisis en la costa caribe del servicio de energía eléctrica prestado por la empresa Electricaribe. Universidad Santo Tomás. Especialización en Derecho Administrativo. Valledupar. Recuperado de https:/ / repository.usta.edu.co/ bitstream/handle/11634/2155/Vencejorge2016.pdf? sequence $=1 \&$ isAllowed $=\mathrm{y}$.

Villegas-Castrillo, E. (1993). El Trabajador Social en el trabajo de grupo. Alternativas. Cuadernos de Trabajo Social, (2), 119-133. doi: 10.14198/ALTERN1993.2.7. 


\section{OTROS ARTÍCULOS DE PROSPECTIVA No. 33}

\section{EDITORIAL}

El Paro Nacional en Colombia 2021: explosión social entre dinámicas estructurales y de coyuntura. Relevancia de la acción política y del diálogo en su desarrollo y transformación Adolfo Adrián Álvarez-Rodríguez

\section{ARTÍCULOS}

Organizaciones sociales de pobladores y prácticas de resistencia en contexto de pandemia COVID-19 en Chile

Patricia Castañeda-Meneses

Entre el aislamiento y las brechas digitales: sistematización de experiencia de acompañamiento socioemocional en personas mayores de Temuco, Chile, en tiempos de COVID-19

G. Bernarda Aedo-Neira

Percepción de apoyo social y calidad de vida: la visión de personas mayores chilenas en el contexto de pandemia durante el 2020

Claudia Elena Quiroga-Sanzana

Gabriela Rocío Parra-Monje

Camila Julia Moyano-Sepúlveda

Marco Alejandro Díaz-Bravo

A propósito del qué, cómo y para qué investigar en el campo de la justicia juvenil: La trastienda de un proceso de investigación en Rosario, Argentina Karina De Bella

Proyección social: reflexión sobre una experiencia virtual con adolescentes en época de pandemia en Sucre y Bolivar, Colombia

Claudia Yaneth Martínez-Mina
La intervención social mediante proyectos comunitarios en modalidad virtual en Mérida, México. Una perspectiva desde el Trabajo Social Claudia Isabel Tzec-Puch Amairani Aracelly Ceh-Alvarado Yanet Guadalupe González-Canul

Resignificación de los vínculos Sociales durante la pandemia de COVID-19: narrativas de personas que convergen en Conjuntos residenciales en Bogotá, Colombia

Andrea Marcela Reyes-García

Laura Daniela Molina-Ortiz

La paradoja de la familia doblemente recluida: clínica de una pandemia en Colombia

Norman Darío Moreno-Carmona

Juan José Cleves-Valencia

Ciencias sociales, mundo y pandemia: por un futuro posible

José Miguel Segura-Gutiérrez

Lina Paola Vásquez-Ávila

¿Qué es Trabajo Social? Los primeros vínculos de los estudiantes con la profesión: experiencias de Argentina, Brasil, Paraguay y Uruguay

Silvia Orieta Rivero-Rodríguez

Leonel Del Prado

Nidia Graciela Battilana-Amarilla

Rosilaine Coradini-Guilherme

Intervención profesional de trabajadores sociales de la universidad del Quindio, Colombia. Asuntos epistemológicos, metodológicos y ético-políticos

Ana María Gil-Ríos 
Reflexiones sobre la intervención social en lo rural: experiencias en el Magdalena Medio, Colombia

Claudia Milena Quijano-Mejía

Johana Linares-García

Construcción de rutas de atención integral a la convivencia escolar en Cali, Colombia: sistematización de la experiencia

Leidy Johana Prado-Montaño

Luz Helena López-Rodríguez

Alejandra Gutiérrez-Cárdenas

Experiencia del proceso de intervención familiar de los actores participantes en la Escuela para Familias del ICBF-Jamundí, Colombia Katherin Viviana Silva-Minotta

María José Forero-Izquierdo

Jimena del Pilar Jaramillo-Jaramillo

\section{RESEÑAS DE LIBROS}

Alerta global: políticas, movimientos sociales y futuros en disputa en tiempos de pandemia Jairo Crispín

El neoliberalismo como teología política. Habermas, Foucault, Dardot, Laval y la historia del capitalismo contemporáneo

José Francisco Desentis-Torres

La Reconceptualización del Trabajo Social en Colombia: Análisis histórico-crítico de las décadas de 1960-1970

Juan Pablo Sierra-Tapiro

ARTISTA INVITADO

Juan Camilo González

Prospectiva

\section{PROSPECTIVA}

Revista de Trabajo Social e Intervención Social

No. 33 • ene.-jun. 2022

e-ISSN: 2389-993X • Universidad del Valle 